Archives

$00 \mid 1988$

Journée d'études Werner Sombart

\title{
La conception du capitalisme chez Braudel et Sombart. Convergences et divergences
}

\section{Wolfgang Mager}

\section{OpenEdition}

\section{Journals}

Édition électronique

URL : http://journals.openedition.org/ccrh/2983

DOI : $10.4000 /$ ccrh.2983

ISSN : 1760-7906

\section{Éditeur}

Centre de recherches historiques - EHESS

Édition imprimée

Date de publication : 14 mars 1988

ISSN : 0990-9141

Référence électronique

Wolfgang Mager, « La conception du capitalisme chez Braudel et Sombart. Convergences et divergences ", Les Cahiers du Centre de Recherches Historiques [En ligne], 00 | 1988, mis en ligne le 13 avril 2009, consulté le 19 avril 2019. URL : http://journals.openedition.org/ccrh/2983 ; DOI : 10.4000/ ccrh.2983

Ce document a été généré automatiquement le 19 avril 2019

(c) Tous droits réservés 


\title{
La conception du capitalisme chez Braudel et Sombart. Convergences et divergences
}

\author{
Wolfgang Mager
}

1 C'est à la grande trilogie de Fernand Braudel sur Civilisation matérielle, économie et capitalisme, $X V^{\mathrm{e}}-\mathrm{XVIII}{ }^{\mathrm{e}}$ siècle, publiée en 1979 et centrée autour de la problématique de la nature et de l'évolution du capitalisme, que je voudrais me référer ici, ainsi qu'aux trois conférences faites à l'Université de Johns Hopkins en 1977 - et publiées d'abord sous le titre Afterthoughts on Material Civilization and Capitalism -, où l'auteur présentait déjà les grandes lignes de son ouvrage.

2 Le titre italien de ces conférences, La Dinamica del Capitalismo, puis celui de La dynamique du capitalisme pour le texte original français paru en 1985, montrent bien la perspective choisie par Braudel dans la trilogie de 1979 pour étudier l'histoire économique et sociale de l'Europe aux temps modernes. Dans son vaste tableau en trois volets de l'histoire moderne ainsi que dans la version abrégée, Braudel développe l'idée que, dans l'évolution $\mathrm{du}$ capitalisme, il faut distinguer deux phases : la première allant du bas Moyen Age au $\mathrm{XVIII}^{\mathrm{e}}$ siècle, suivie d'une seconde phase datant de la révolution industrielle. Comme on le voit, l'auteur tend à relativiser l'importance de la grande industrie dans la longue histoire du capitalisme. En outre, il met en doute le caractère exceptionnel des inventions et innovations intervenues depuis le XVIII ${ }^{\mathrm{e}}$ siècle. En se rapportant à John U. Nef, selon lequel une première révolution anglaise serait survenue entre 1560 et 1640, Braudel note : " Disons que la révolution anglaise qui s'affirmera au XVIII ${ }^{\mathrm{e}}$ siècle a déjà commencé au XVI , qu'elle a progressé par paliers ». De cette observation, Braudel passe à une hypothèse plus générale: "Ne peut-on en dire autant de l'Europe où, depuis le $\mathrm{XI}^{\mathrm{e}}$ siècle, les expériences se succèdent, se relient entre elles et en quelque sorte s'accumulent?» Selon Braudel, «tour à tour, chaque région, à une époque ou à une autre, a connu des poussées préindustrielles, avec les accompagnements que cela implique, en particulier sur le plan 
de l'agriculture. » L'auteur en arrive à la conclusion : l'industrialisation a été « endémique à travers le continent » depuis le haut Moyen Age.

3 Si Braudel place son histoire économique et sociale de l'époque moderne sous le concept du capitalisme, il le fait en se rapportant expressément à Sombart. Braudel rappelle que la notion de capitalisme, "dans son usage large, date du début même du $\mathrm{xx}^{\mathrm{e}}$ siècle ». «En fait, ce n'est qu'au début de notre siècle qu'il jaillit dans sa pleine force des discussions politiques, comme l'antonyme naturel de socialisme. Il sera lancé dans les milieux scientifiques par le livre éclatant de W. Sombart, Der moderne Kapitalismus (1ère édition, 1902). "Dans l'avant-propos du tome 3 de la trilogie, Braudel n'hésite pas à parler en termes emphatiques de "l'ouvrage monumental de Werner Sombart, Der moderne Kapitalismus (dernière édition, 1928) ", qu'il qualifie de "somme fantastique de lectures et de mises au point ». A maints endroits de son ouvrage, Braudel fait référence à l'opus magnum de Sombart. Dans l'index, son nom est attesté pas moins de 37 fois, seulement dépassé par celui de Karl Marx, cité 42 fois. Braudel a profité autant des conceptions de Sombart sur la nature et la dynamique du capitalisme que de la richesse matérielle d'un ouvrage « assorti d'une somme exubérante de preuves ». Dans un compte rendu de 1959 du livre d'Otto Brunner Neue Wege der Sozialgeschichte, Braudel a nommé Sombart parmi ses "vieux compagnons de lecture ", en le plaçant en tête d'une liste de six auteurs allemands comprenant également Max Weber, Friedrich Meinecke, Heinrich Mitteis, Otto Hintze et Theodor Mayer. Dans son étude Histoire et sociologie qui fait partie du Traité de sociologie, édité par Georges Gurvitch (t. 1, 1958), Braudel, en traitant de l'intérêt novateur des historiens pour la psychologie, cite à la fois Sombart et Lucien Febvre. Au dire de Braudel, Werner Sombart était le précurseur - c'est l'époque où Werner Sombart affirme que le capitalisme est d'abord esprit »-, et Braudel d'ajouter : «Bien plus tard, toujours dans cette même ligne de conquête, Lucien Febvre parlera d'outillage mental ». Si l'on se rappelle l'admiration et l'affection de Braudel pour Lucien Febvre, le rapprochement de Sombart avec Febvre prend toute sa signification.

Dans ce qui suit, je me propose de comparer les approches de Braudel et Sombart dans leur compréhension du capitalisme, en évoquant rapidement leurs idées maîtresses sur la nature et la dynamique du capitalisme, avant de les confronter.

Braudel, dans Civilisation matérielle, économie et capitalisme, développe une "tripartition " de l'économie, un «schéma tripartite » comme «table de référence » de son ouvrage; il parle de «trois étages » de l'économie. Au bas de cette hiérarchie, au-dessous du marché, il constate une "économie hors marché » ou "infra-économie », constituée par des "paysans dans leurs villages qui vivent de façon presque autonome, quasi en autarcie ». Il s'agit du champ de «l'autoconsommation » ou de «l'autosuffisance », complétée par le "troc des produits et des services». Cette "activité élémentaire de base que l'on rencontre partout et qui est d'un volume tout simplement fantastique » est désignée par Braudel - «faute de mieux»-comme "vie matérielle» ou "civilisation matérielle ». Braudel nous la présente dans le premier volume de sa trilogie de 1979 sous le titre Les structures du quotidien: le possible et l'impossible. Cette dernière tournure-le possible et l'impossible-se rapporte aux étroites limites de ce que les hommes d'hier pouvaient atteindre par leur travail. Au-dessus de la civilisation matérielle se retrouve, selon Braudel, «l'économie dite de marché ». L'auteur entend par là «les mécanismes de la production et de l'échange liés aux activités rurales, aux échoppes, aux ateliers, aux boutiques, aux bourses, aux banques, aux foires et naturellement aux marchés ». Ce qui caractérise l'économie de marché, c'est l'équilibre entre les partenaires participant à 
l'échange, c'est la concurrence transparente, reconnaissable à « la fluctuation et l'unisson des prix entre les marchés d'une zone donnée » « selon l'offre et la demande », indicateur d'un échange égal. Prennent part à l'économie de marché, à côté des artisans, les paysans, dans la mesure où ils fournissent des produits au marché : «Le paysan, qui lui-même commercialise régulièrement des outils, des vêtements, fait déjà partie du marché. Celui qui ne vient au bourg que pour vendre quelques menues marchandises, des oeufs, une volaille, pour obtenir les quelques pièces de monnaie nécessaires au paiement de ses impôts ou à l'achat d'un soc de charrue, celui-là touche seulement à la limite du marché. Il reste dans l'énorme masse de l'autoconsommation. »C'est au-dessus du marché fourni par les petits producteurs et consommateurs que se situe, selon Braudel, le champ du capitalisme. Il est vrai que les capitalistes prennent part à l'échange. Cependant, à la différence des petits producteurs de valeurs d'usage, les capitalistes tentent de réaliser un profit. Ils le font en imposant un échange inégal, par le moyen de monopoles, de cartels, de privilèges de droit ou de fait, qui témoignent de la supériorité sociale, politique et culturelle des capitalistes sur les autres groupes de la société. C'est ainsi que le capitalisme est "privilège du petit nombre", "accumulation de puissance (qui fonde l'échange sur un rapport de force autant et plus que sur la réciprocité des besoins) ». Son domaine propre, à l'époque moderne, c'est le commerce, et, en tout premier lieu, le commerce au loin, son champ d'action portant sur la circulation des biens, et moins sur leur production. «L'action capitaliste », c'est avant tout l'affaire des négociants. Au cours des Journées Fernand Braudel, qui se sont déroulées à Châteauvallon en octobre 1985, Braudel a précisé: «Le capitalisme est un phénomène de superstructure, c'est un phénomène de minorité, c'est un phénomène d'altitude. Chaque fois que j'ai étudié les grands capitalistes-marchands, banquiers-j'ai été stupéfait de les voir aussi peu nombreux ». «L'avantage et la supériorité du capitalisme, c'est la possibilité de choix ». «Un monopole vous abandonne? Eh bien on en trouve un autre. C'est la mort du capitalisme du grand-père et du père, mais pas du capitalisme du fils ou du petit-fils ».

6 Les idées de Braudel sur la nature de l'économie de marché ainsi que sur le capitalisme ont été développées par l'auteur dans le troisième volume de sa trilogie de 1979, auquel il a donné le titre Les jeux de l'échange.

7 Quant au rapport entre les trois étages de son schéma tripartite, entre l'autosuffisance paysanne, le marché des petits producteurs et le champ d'action des capitalistes, Braudel plaide en faveur d'un engrenage dialectique. "On a souvent présenté le capitalisme comme le moteur ou l'épanouissement du progrès économique ", remarque l'auteur, pour affirmer pour sa part : «Je crois obstinément que c'est le mouvement d'ensemble qui est déterminant et que tout capitalisme est à la mesure, en premier lieu, des économies qui lui sont sous-jacentes».

8 L'évolution du capitalisme, sa dynamique à l'époque moderne est liée, selon Braudel, à son insertion dans ce qu'il appelle économie-monde, en adaptant par ce néologisme le terme allemand de Weltwirtschaft. La présentation des diverses économies-mondes qui se sont succédées en Europe depuis le haut Moyen Age est le sujet du troisième volume de la trilogie de 1979, intitulé Le temps du monde. A l'élaboration d'un modèle structural des diverses dimensions de l'économie de l'Europe aux temps modernes fait suite le modèle des processus de la dynamique du capitalisme. Celle-ci se réalise, selon Braudel, dans la séquence d'économies-mondes. Que faut-il entendre par là?

9 Le concept d'économie-monde, tel qu'il est utilisé dans Civilisation matérielle, économie et capitalisme, est lié à un concept analogue que Braudel avait développé dès sa thèse sur $L a$ 
Méditerranée et le monde méditerranéen à l'époque de Philippe II, dont la première édition date de 1949. Mettant à profit des auteurs comme Fritz Rörig (Mittelalterliche Weltwirtschaft : Blüte and Ende einer Weltwirtschaft, 1933), Braudel avait alors étudié l'espace méditérranéen du point de vue de la «répartition plus ou moins parfaite de l'espace en zones économiques complémentaires, liées les unes aux autres, hiérarchisées, dans un ordre géographiquement dessiné », en adoptant la perspective de "ce que les Allemands appellent une Weltwirtschaft, pour conclure que la Méditerranée, au XVI ${ }^{e}$ siècle, en est une à elle seule ». Il constate l'existence d'une économie-monde méditerranéenne dominée par les villes-États de l'Italie du Nord. Dans la seconde édition de son ouvrage, publiée en 1966, l'auteur précise : «Ce monde d'une soixantaine de jours est bien, grosso modo, une Weltwirtschaft, une économie-monde, un univers en soi. Tout n'y est pas ordonné de façon stricte et autoritaire, mais les grandes lignes d'un ordre s'y dessinent. Ainsi toute économie-monde accepte un centre, une région décisive qui donne l'impulsion aux autres et établit, à elle seule, l'unité qui est en cause. De toute évidence, ce centre méditerranéen, au $\mathrm{XVI}^{\mathrm{e}}$ comme au $\mathrm{Xv}^{\mathrm{e}}$ siècle, est un étroit quadrilatère urbain, Venise, Milan, Gênes, Florence, avec ses discordances, ses rivalités de ville à ville, chacune $\mathrm{y}$ pesant d'un poids variable. " Dans la première édition de La Méditerranée, Braudel ne traite guère la thématique du capitalisme. Par contre, dans la deuxième édition, il place explicitement l'évolution de l'économie-monde méditerranéene sous l'idée directrice du capitalisme et arrive à la conclusion: «Il n'est... pas abusif, dans nos classifications, de parler d'un capitalisme marchand en désignant ainsi une forme agile, déjà moderne et sûrement efficace de la vie économique du siècle. Tout n'aboutit pas à ses prouesses, mais bien des choses dépendent de son dynamisme et de son attraction. Les impératifs du grand commerce, du commerce à longue distance, ses accumulations de capital, jouent un rôle moteur ".

Braudel a repris, en le dynamisant, le concept d'économie-monde dans le tome 3 de Civilisation matérielle, économie et capitalisme. Dans cet ouvrage, il conçoit l'histoire du capitalisme européen comme une séquence de centrages, de décentrages et de recentrages d'économies-mondes sous la domination d'abord de Venise (à partir de 1380), ensuite d'Anvers (à partir de 1500 environ), puis de Gênes (à partir de 1550-60), et d'Amsterdam (à partir de 1590-1610), finalement de Londres depuis 1780-1815, jusqu'à ce que New York prît la place de Londres (en 1929). Braudel précise, en 1979, le concept d'économie-monde "comme une triple réalité ». «Elle occupe un espace géographique donné ", elle "accepte toujours un pôle, un centre, représenté par une ville dominante, jadis un État-ville, aujourd'hui une... capitale économique ", " toute économie-monde se partage en zones successives. Le cœur, c'est-à-dire la région qui s'étend autour du centre... Puis viennent des zones intermédiaires, autour du pivot central. Enfin, très larges, des marges qui, dans la division du travail qui caractérise l'économie-monde, se trouvent subordonnées et dépendantes, plus que participantes ». Selon Braudel, la suite des économies-mondes ne constitue pas moins qu'« une histoire profonde du monde ». Au dire de l'auteur, les «économies-mondes successives, bâties sur l'Europe à partir de l'expansion européenne » expliquent « les jeux du capitalisme et sa propre expansion ». Il conclut: «Ces économies-mondes typiques ont été les matrices du capitalisme européen, puis mondial ». La question est de savoir si l'on peut détecter une logique qui ait commandé au déroulement des centrages et des décentrages des économies-mondes européennes. Braudel ne donne pas de réponse satisfaisante à ce problème, si ce n'est que « centrage, décentrage, recentrage semblent liés, d'ordinaire, à des crises prolongées de 
l'économie générale. C'est donc au travers de ces crises qu'il faut, sans doute, aborder l'étude difficile de ces mécanismes d'ensemble par quoi l'histoire générale se retourne ».

11 Quant aux idées principales que Sombart développe sur la nature et l'évolution du capitalisme, je tenterai de les résumer en me référant aussi aux réflexions sur le capitalisme à l'époque moderne qu'il développe dans le deuxième volume de son ouvrage sur le Capitalisme moderne et le sous-titre du tome 2, « La vie économique européenne à la première phase du capitalisme, particulièrement $a u \mathrm{XVI}^{\mathrm{e}}, \mathrm{XVII}^{\mathrm{e}}$ et $\mathrm{XVIII}^{\mathrm{e}}$ siècle ». Tout comme Braudel, Sombart pense qu'à l'époque moderne la vie économique a été déterminée par le jeu de trois systèmes économiques : " $1^{\circ} l^{\prime}$ économie domestique sous

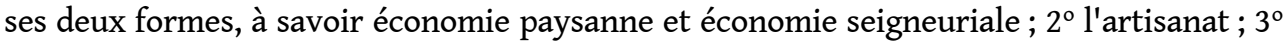
le capitalisme ", chacun de ces systèmes étant déterminé à la fois par : une organisation spécifique de la production; une attitude ou un esprit économique particulier ( Wirtschaftsgesinnung, Wirtschaftsprinzip); et enfin une technique de travail appropriée. L'économie domestique repose sur l'exploitation paysanne : les terres appartiennent à un agriculteur qui repose sur les travaille avec l'aide de sa famille. L'esprit de l'économie domestique, c'est l'autosuffisance des paysans et des seigneurs; sa technique de travail consiste en procédés empiriques et traditionnels. Par artisanat, Sombart entend l'économie d'échange non capitaliste qui est fondée sur la division du travail entre petits producteurs agricoles et industriels qui vendent leurs produits au marché, le plus souvent sans l'intervention de marchands ou commerçants. Le principe économique est ici l'approvisionnement des agriculteurs en produits industriels et des artisans en produits agricoles, et la commercialisation des produits a donc lieu en vue de fournir des biens d'usage. La technique de travail reste empirique et traditionnelle. Quant au système capitaliste, il repose sur l'entreprise qui forme une entité en soi, séparée de la personne de son propriétaire. Trois aspects caractérisent l'entreprise : elle est unité juridique, unité 'de comptabilité et unité de crédit (Rechtseinheit, Rechnungseinheit, Krediteinheit). «L'émancipation de la firme de la personne de son propriétaire », c'est «l'essence même de l'entreprise capitaliste ». L'esprit capitaliste, c'est la mise en valeur du capital investi dans l'entreprise, c'est donc la recherche du profit et de son accumulation. A ce dynamisme inné du capitalisme correspond une technique de travail rationnelle qui s'est réalisée de façon classique, au dire de Sombart, dans la comptabilité en partie double. «Concevoir le capitalisme sans comptabilité en partie double, c'est totalement impossible : l'un est à l'autre comme la forme au contenu ».

12 Dans son livre Der moderne Kapitalismus, Sombart a examiné « les étapes de la genèse du capitalisme à partir des formes économiques du moyen âge européen ». Comme Braudel, Sombart refuse la théorie de la succession des systèmes économiques en phases, en stades, la soi-disant Stufentheorie de la croissance économique. Le passage de l'économie domestique à l'économie d'échange et de celle-ci à l'économie capitaliste est conçu, dans Der moderne Kapitalismus, comme engrenage et téléscopage de trois plans économiques. Sombart distingue dans l'évolution du capitalisme l'aube du capitalisme, le capitalisme à son apogée et le capitalisme tardif (Früh-, Hoch-, Spätkapitalismus). L'économie d'échange et l'économie domestique ont continué d'être largement pratiquées pendant la première phase du capitalisme, remarque l'auteur. "Partout on observe des éléments des anciens systèmes économiques, qui se sont maintenus. C'est ainsi que l'économie domestique et l'économie artisanale se trouvent à côté de systèmes économiques modernes, en pleine évolution, tel que le capitalisme ». Sombart constate : «A la fin de la première époque du capitalisme, l'agriculture était pour ainsi dire indemne de l'impact capitaliste. Avec la 
seule exception du gros négoce et de quelques branches du commerce, l'intrusion du capitalisme dans l'organisation traditionnelle de l'économie est restée marginale. On insistera sur la résistance de l'artisanat." Sombart met en relief, comme traits caractéristiques du capitalisme à la fin de sa première époque les points suivants : «Une exploitation considérable de peuples lointains, un commerce important de marchandises lié à l'exploitation de ces peuples, un système de crédit développé, un monde bancaire en plein épanouissement, des finances publiques florissantes aux mains de traitants; enfin les débuts modestes d'une grande industrie concentrée. » Il apparaît donc que Braudel et Sombart ont des idées assez proches sur le capitalisme. Les deux auteurs font remonter la genèse du capitalisme à l'époque de la Renaissance italienne. Tous deux préconisent également un modèle tripartite de l'économie en distinguant entre l'économie domestique autosuffisante, l'économie de petits producteurs échangeant des biens d'usage et l'économie capitaliste, reliées entre elles par un rapport dialectique. Pour ces deux auteurs, la source principale du profit capitaliste à l'époque moderne ne résidait pas dans la production des biens industriels mais dans leur circulation: les capitalismes marchand et financier l'emportaient donc largement sur le capitalisme industriel. Enfin, ils sont convaincus qu'à l'époque moderne les profits étaient surtout tirés de l'exploitation de peuples lointains et d'échanges inégaux.

Néanmoins, Braudel et Sombart divergent sur un point important, à savoir l'explication de la dynamique du capitalisme moderne. On sait que pour Braudel, la dynamique du capitalisme repose sur ce qu'il appelle l'histoire profonde du monde, c'est-à-dire sur l'intervalle entre les économies-mondes et les temps modernes. Sombart n'a pas approfondi lui-même ce modèle, qu'il esquisse dans l'avant-propos du troisième volume de Der moderne Kapitalismus en traitant de l'essor de l'Angleterre au XVIII ${ }^{\mathrm{e}}$ siècle. Il y note que le destin du capitalisme est lié au fait « qu'il a développé, à un endroit minuscule de la surface mondiale, ses formes les plus poussées, pour dominer le monde entier. Pour arriver à un peu de clarté dans le chaos des événements innombrables de l'histoire, il faut distinguer entre un centre capitaliste, des pays capitalistes centraux et des pays qu'on considérera comme périphériques par rapport à ce centre; les pays centraux sont les nations actives et dominantes, les pays périphériques les nations passives, servantes ». Sombart applique cette réflexion à la révolution industrielle en Angleterre, en Europe occidentale et à l'est des États-Unis, et non pas à l'histoire de la première époque du capitalisme. Il en explique la dynamique par le potentiel dynamisant de l'esprit capitaliste, c'est-à-dire la combinaison de la volonté de gain avec des vertus bourgeoises telles que «application au travail, modération, goût de l'épargne, sens de la rentabilité, fidélité aux contrats", et il affirme: «L'état d'esprit né de l'intégration de l'esprit bourgeois, c'est l'esprit capitaliste. C'est lui qui a créé le capitalisme ». Braudel ne peut tout à fait accepter l'idée de déduire le capitalisme de la volonté de puissance et du rationalisme des bourgeois et questionne : « Faut-il... attribuer à nos acteurs un esprit qui serait la source de leur supériorité et les caractériserait une fois pour toutes, qui serait calcul, raison, logique, détachement des sentiments ordinaires, le tout au service d'un appât effréné du gain ?» Par ailleurs, il constate : «Cette opinion passionnée de Sombart a perdu beaucoup de sa crédibilité » et refuse une «explication idéaliste... qui fait du capitalisme l'incarnation d'une certaine mentalité ». Cette explication aurait été «la porte de sortie qu'empruntèrent, faute d'une autre, Werner Sombart et Max Weber pour échapper à la pensée de Marx ». Braudel rejette cette idée de déduire le capitalisme du " progrès de l'esprit scientifique et de la rationalité, au coeur de l'Occident, qui auraient assuré l'essor économique général de l'Europe, portant en avant sur leur propre 
mouvement le capitalisme, ou mieux l'intelligence capitaliste et sa percée constructive. C'est faire la part du lion à l'esprit, aux innovations des entrepreneurs ». Braudel ne parle pas sans ironie du « lyrisme » avec lequel Sombart a célébré « l'utilité de la partita doppia ", de la comptabilité en partie double. "Sombart est allé outre et sur son élan, d'autres exagéreront à leur tour ».

Enfin, il faudrait aussi insister sur une autre idée commune à Braudel et Sombart, mais cette fois sur le capitalisme tout court, comprenant donc également l'époque contemporaine et l'histoire des économies-mondes capitalistes en dehors de l'Europe. Selon Braudel, il existait à l'époque moderne à côté du capitalisme européen d'autres économies-mondes analogues en Chine, au Japon, en Inde, dans les pays islamiques et ailleurs. Mais ce qui, pour lui, est caractéristique de l'évolution du capitalisme en Europe, c'est le transfert du centre capitaliste d'Amsterdam à Londres, parce que l'avènement de Londres allait de pair avec la création d'une économie nationale capable de dominer le monde entier. Sous la domination de Londres et de l'Angleterre, l'économie-monde européenne se transforma en économie mondiale. «Pour la première fois, l'économie mondiale européenne, bousculant les autres, va prétendre dominer l'économie mondiale et s'identifier avec elle à travers un univers où tout obstacle s'effacera devant l'Anglais, lui d'abord, mais aussi devant l'Européen ».

Cette thèse d'un capitalisme unique européen, après la " conquête de l'univers " par les Anglais sous le signe de la paix britannique, est à rapprocher des propos tenus par Sombart. A la fin du deuxième volume de Der moderne Kapitalismus, celui-ci constate que l'évolution du capitalisme européen était menacée d'arrêt parce que l'exploitation des peuples de la périphérie était pour ainsi dire achevée. Et Sombart de conclure: «L'évolution de la civilisation matérielle de l'Europe était manifestement arrivée à un point que toutes les autres civilisations elles aussi, avaient atteint à un moment donné, sans pouvoir passer outre. Jetons un coup d'oeil sur l'évolution de la Chine et de l'Inde, de l'Egypte ou de Babylone, de la Grèce ou de Rome : partout nous constatons qu'à la fin ils étaient acculés à la même impasse que l'Europe à la fin de la première époque du capitalisme ». Dans tous les cas cités, le capitalisme s'est écroulé. Par contre en Europe, il s'est frayé un chemin unique au monde, et c'est ce qui fait sa spécificité.

Je me suis efforcé de montrer la grande influence exercée par Sombart, avec ses modèles économiques et la richesse de ses matériaux, ce dont Braudel était le premier à convenir. Ce qui fait l'originalité de l'historien français, c'est d'abord sa théorie des économiesmondes et de leurs séquences à l'époque moderne et contemporaine, et ce qui le distingue principalement de Werner Sombart est le caractère littéraire de son oeuvre. Le professeur allemand a fait du capitalisme un phénomène unique (un historisches Individuum), auquel l'académicien français a insufflé âme et vie. 